\title{
The Effect of Some Meteorological Parameters on the Cosmic Ray Muons detected by KACST detector
}

\author{
Maghrabi A. $\mathbf{H}^{\mathbf{1}}$ \\ National Centre for Applied Physics, King Abdulaziz City for Science and Technology, \\ Address, P.O.Box 6086Riyadh 11442, Saudi Arabia. \\ Email:amaghrabi@kacst.edu.sa

\section{Aldosary A.F} \\ National Centre for Applied Physics, King Abdulaziz City for Science and Technology, \\ Address, P.O.Box 6086Riyadh 11442, Saudi Arabia. \\ Email:aaldosary@kacst.edu.sa
}

\begin{abstract}
To study the cosmic ray modulation properly, it is important to remove atmospheric effects. In this study cosmic ray data from the KACST detector in Riyadh, Saudi Arabia $(\mathrm{Rc}=14.4 \mathrm{GV})$, for the period 2007-2009, were used to study the effect of some meteorological parameters on cosmic ray muons. The parameters of interest are the atmospheric pressure, temperature, and relative humidity. We have found that the atmospheric pressure and temperature are inversely correlated with the muon rate. On the other hand, the relative humidity is correlated with the number of cosmic ray muons.
\end{abstract}


The Effect of Some Meteorological Parameters on the Cosmic Ray Muons detected by KACST detector

35th International Cosmic Ray Conference - ICRC217-

10-20 July, 2017

Bexco, Busan, Korea 


\section{Introduction}

Solar activity modulates the primary cosmic ray particles on different time scales [1]. This modulation leads to changes in some atmospheric properties, which may influence global weather and climate [2-3]. Primary cosmic rays collide with atmospheric molecules, producing a cascade of secondary particles which are detectable by ground level cosmic ray detectors. During their flight in the atmosphere, these secondary particles are affected by atmospheric instabilities and changes. At the detection level, the majority of the observed particles are muons. Their rate depends on several meteorological factors such as the atmospheric pressure, temperature, and the height of the pion production level (height of the first interaction); see for example [4-7]. Several investigations have shown that the variations in the atmospheric variables' coefficients depend on different factors, such as the type of detected particle and the cutoff rigidity of the considered site [8-9].

In order to study the properties of the primary cosmic rays and their modulations caused by the solar and heliospheric instabilities, corrections to the secondary cosmic ray particles, due to atmospheric influences, must be made as in Dorman [4] and Maghrabi and Almutayri[7] for example. The main aim of the current study is to investigate experimentally the relationship between the cosmic ray muons detected by the King Abdulaziz City for Science and Technology (KACST) detector, and the atmospheric pressure, temperature, and relative humidity, and to obtain the correction coefficients of these variables.

\section{Instrumentation and Methods}

In 2002 the KACST installed and began operation of a single channel muon detector (Riyadh latitude $24^{\circ} 43^{\prime}$, longitude 46 40', altitude $613 \mathrm{~m}$, and $\mathrm{Rc}=14 \mathrm{GeV}$ Saudi Arabia) for cosmic ray variation studies. The details of this detector and its calibration procedures can be found in several papers [10-11].

The detector basically consists of a $1000 \mathrm{~mm} \times 1000 \mathrm{~mm} \times 50 \mathrm{~mm}$ sheet of plastic scintillator, contained in a light-tight box with a photomultiplier tube (PMT) placed at the centre of the box to view the scintillation. The signals from the PMT are first selected from the background PMT noise using a discriminator. The selected signals are then amplified using a commercial preamplifier and amplifier circuits. An analogue-to-digital converter (ADC) circuit has been developed [10], and is used to digitize the amplified signal from the PMT. The data were acquired every 15 minutes, along with the atmospheric pressure and room temperature. In this study, daily averages of the cosmic ray muons data for the solar quiet period (2007-2009) have been used.

The rate of the cosmic ray muons due to changes in a meteorological variable $\mathrm{x}$, can be determined experimentally by [6-7]:

$$
\frac{\mathrm{I}_{\mathrm{i}}-\mathrm{I}_{0}}{\mathrm{I}_{0}}=\alpha\left(\mathrm{x}_{\mathrm{i}}-\mathrm{x}_{0}\right)
$$


where $I_{0}$ is the mean muon intensity, $x_{0}$ is the mean value of the $x$ variable during the considered time period, and $\alpha$ is the coefficient of the corresponding variable, which is obtained from the fit between the measured muon rates and that variable.

Due to its important role in affecting all of the cosmic ray secondary particles, the relationship between atmospheric pressure and the muon rate was investigated first. The resultant pressure coefficient was then used to remove this effect from the measured data. Pressure corrected muon data were then correlated independently with the air temperature and relative atmospheric humidly, in order to obtain the correction factors due to the changes in these two variables. The temperature and relative humidity coefficients were then used to remove the effects of these variables from the muon rates.

\section{Results and Discussion}

\subsection{Pressure Effect}

Figure (1) indicates the daily mean values of the muon rate plotted against the atmospheric pressure during the study period. Although of the existence of some scatter in the data, it is obvious that the muon rate decreases as the atmospheric pressure increases [9 and12]. This suggests that as the atmospheric masses (atmospheric pressure) above the detector at certain ground level increases the number of secondary muons will be decreased. Regression analysis between the two variables gives a slope (the barometric coefficient) of $0.11+0.003 \% / \mathrm{mbar}$ with a 0.77 correlation coefficient (r). The obtained barometric coefficient was then used to correct the muon rate for the pressure effect using Equation (1).

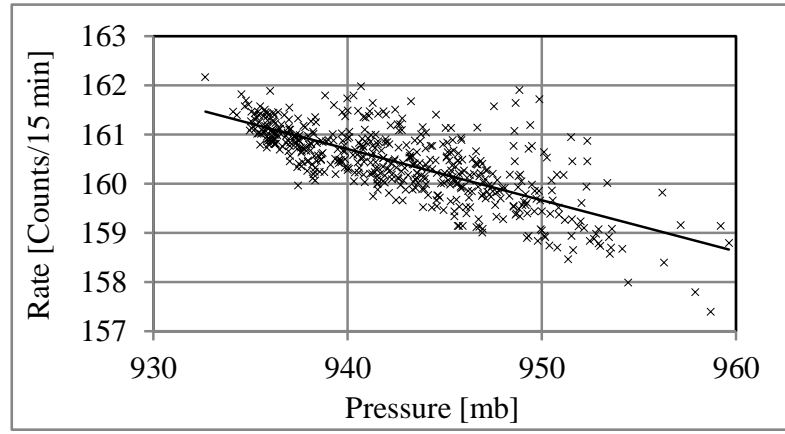

Figure 1. Scatter plot of the daily values of atmospheric pressure and the muon count rate for the period 2007-2009. The straight line is the best linear fit to the data where the slope is the barometric coefficient.

Figure (2) is a scatter plot of the muon rate versus atmospheric pressure after removing the pressure effect. It is

clear that the relationship between the two variables has been reversed. Although the pressure influence has been removed, there is still a remaining residual effect with a value of $0.024 \%$ /mbar (about $24 \%$ of the barometric coefficient) and correlation coefficient $(r)=0.44$. This residual value is expected to be insignificant in some cosmic ray investigations [13]. However, it may be needed to be taken into account for certain studies such as the influence of cosmic rays on climate [3].

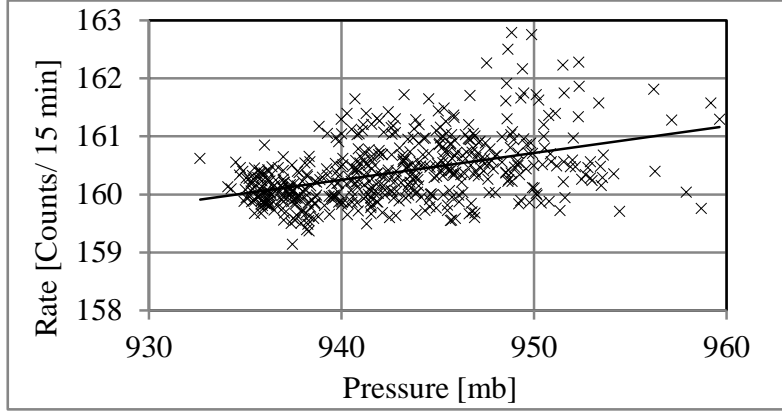

Figure 2. As Figure (1) except that, here, the pressure corrected muon rate is plotted against the atmospheric pressure. The slope of the least square fit to this data gives the remaining residual of the pressure effect. 
Figure (3) is a time series showing the variations of the monthly averages of the raw muon rate, the atmospheric pressure, and the pressure corrected muon rate, during the study period. The pressure and the pressure corrected rates attain their minimum values during summer and their maximum in winter time, whereas the raw muon rates show the opposite trend. The average difference between the two muon rates was about $1 \%$. It is noticeable that, when the pressure effect was removed from the cosmic ray muon data, the seasonal variation is presented.

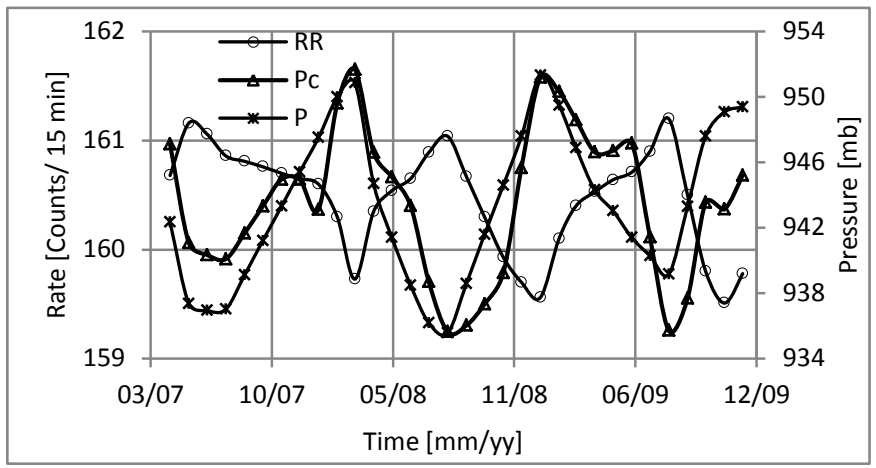

Figure 3.Time series of the one month averages of atmospheric pressure $(\mathrm{P})$, raw muon rate (RR), and the pressure corrected rate $(\mathrm{Pc})$, for the time period between 2007 and 2009.

\subsection{Temperature Effect}

Figure (4) displays the relationship between the pressure corrected muon rates and the air temperature at ground level. It can be seen that the number of cosmic ray muons is reduced as the temperature increases. This anti-correlation between the two variables is in agreement with the previously published results of several researchers [6-7]. The decrease in the muon rate as the temperature increases is due to changes in the atmospheric density, which in turn depends on atmospheric temperature. As the temperature increases, the atmosphere becomes less dense, and the distance between the first interaction and the detection level will be longer. Consequently, muons which are produced will travel a longer distance, which means that higher numbers of them will decay before arriving at the detector. In contrast, during low temperature periods the distance travelled in the atmosphere will be shorter and denser, hence fewer muons will decay.

A least square fit between the pressure corrected muons and the temperature gives a temperature coefficient of $-0.028 \pm 0.002 \% / /{ }^{\circ} \mathrm{C}$ with a correlation coefficient of 0.64 .

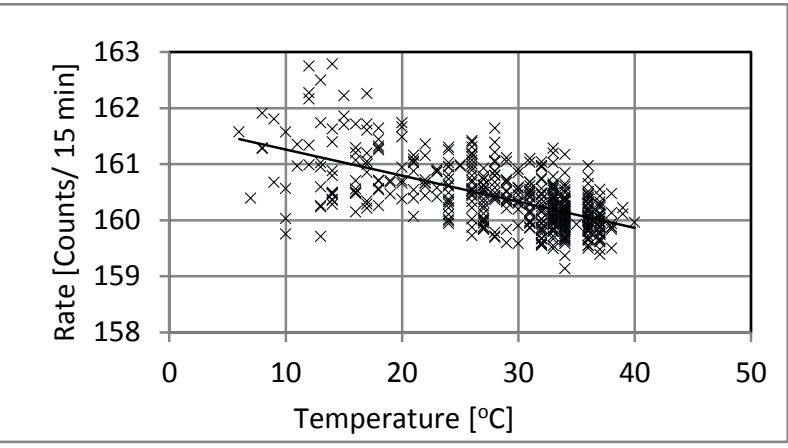

Figure 4. Scatter plot between the daily values of air temperature and the pressure corrected muon count rate for the period 2007-2009. The straight line is the best linear fit to the data, with slope equal to the temperature coefficient.

It is noteworthy that the temperature coefficient calculated for this site is

somewhat smaller than those reported at other locations (for instance [6 and 13]. We expect that this may be due to the high rigidity of the KACST detector's location. This is supported by theoretical investigations conducted [14] who revealed a comparable temperature coefficient value for a site with the same threshold energy as Riydah. 
The temperature residual was calculated using the same approach as for the pressure residual. A significant residual of $0.010 \pm 0.001 \% / /^{\circ} \mathrm{C}$ (about $63 \%$ of the temperature coefficient) with an $\mathrm{r}$ value of 0.3 was found.

Figure (5) compares the monthly mean values of the air temperature, pressure corrected, temperature corrected, and raw muon rates for the period 2007-2009. Although the raw muon rate follows the temperature trends, the two corrected muon rates do not. It can also be seen that the temperature corrected muon rate differs slightly from the pressure corrected one.

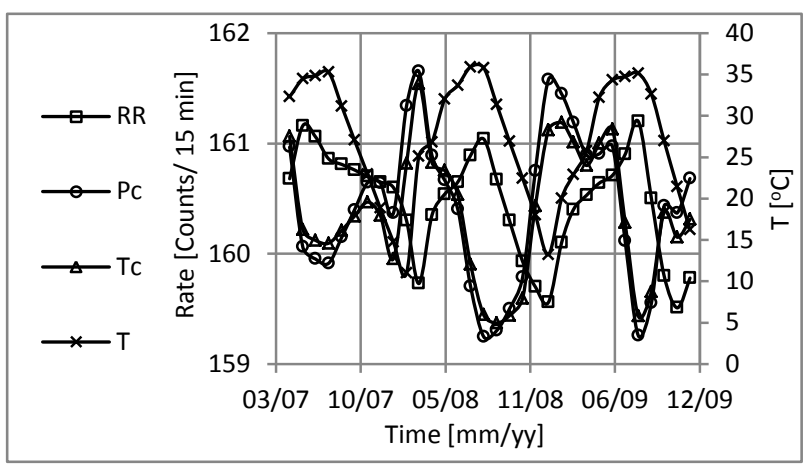

Figure 5.Time series of the one month average temperature $(\mathrm{T})$, raw muon rate $(\mathrm{RR})$, pressure corrected rates $(\mathrm{Pc})$, and temperature corrected rate $(\mathrm{Tc})$ for the period between $2007-2009$.

\subsection{Relative Humidity Effect}

The effect of relative humidity on cosmic ray secondary particles has been investigated less, due to the absence of a relationship between the two variables as suggested by some researchers [4]. However, in this study we aim to explore the possibility that relative humidity has an effect on cosmic ray muons as detected by the KACST detector. The influence of relative humidity on the pressure corrected muon rate is displayed in Figure (6). While some spread in the data exists, it is clear that the two variables are directly related to each other. In other words, the number of muons increases as the relative humidity increases.

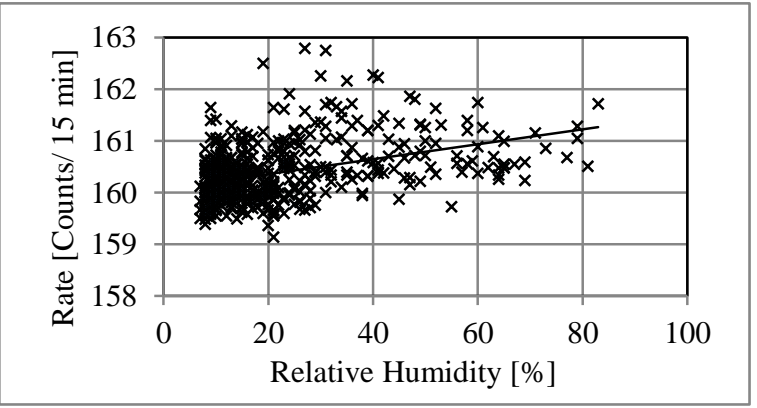

Figure 6. Scatter plot between the daily values of the relative humidity and the pressure corrected muon count rate for the considered period.

The daily values of the pressure corrected muon data were linearly fitted with the relative humidity; a coefficient of +0.010 $\pm 0.0009 \%$ for every $1 \%$ change in the relative humidity was found. The correlation coefficient of the relationship was 0.59 . It is clear that the relative humidity has the same magnitude of effect as the temperature on the cosmic ray muons, although it is different in sign.

Figure (7) compares the monthly mean values of the raw, pressure corrected, temperature corrected, and relative humidity corrected muon rates for the considered period. The data with relative humidity correction did not differ much from those corrected for temperature and pressure effects. 


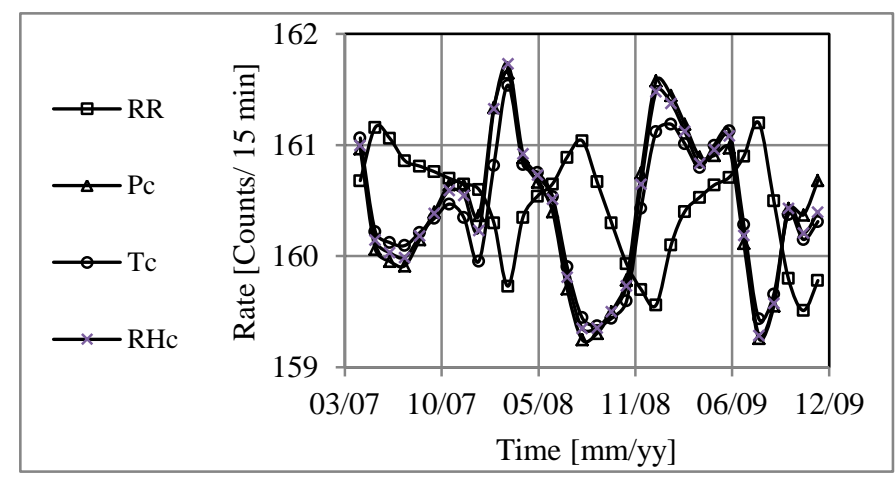

Figure 7. Time series of the one month average raw muon rate(RR), pressure corrected rates $(\mathrm{Pc})$, relative humidity corrected (RHc), and temperature corrected (Tc) rate for the period between 2007 and 2009 .

\subsection{Pressure and Temperature Effect}

Multiple regression analyses have been carried out between the muon rate and both the atmospheric pressure and temperature to obtain the correction coefficients using Duperier method [15]. The barometric and temperature coefficients were $-0.12 \pm 0.004 \% / \mathrm{mbar}$ and -0.047 $\pm 0.0032 \% /{ }^{\circ} \mathrm{C}$, respectively, and the correlation coefficient of this regression was 0.86 . The obtained coefficients were then used to correct for the atmospheric effect. The results of this correction, as well as the raw rate, pressure, and temperature corrected data, are displayed in Figure (8). Remarkably, the corrections made using the first method by considering the pressure and temperature effects separately, follow the same trend and have negative effects on the raw muon count rates. On the other hand, the corrected muon rates obtained using the Duperier method follow the trend of the raw data. Another finding from this graph is that seasonal variations exist even when the corrections were made using different methods. This strongly suggests that information about atmospheric variables at various levels in the atmosphere may be used to remove this seasonality [7].

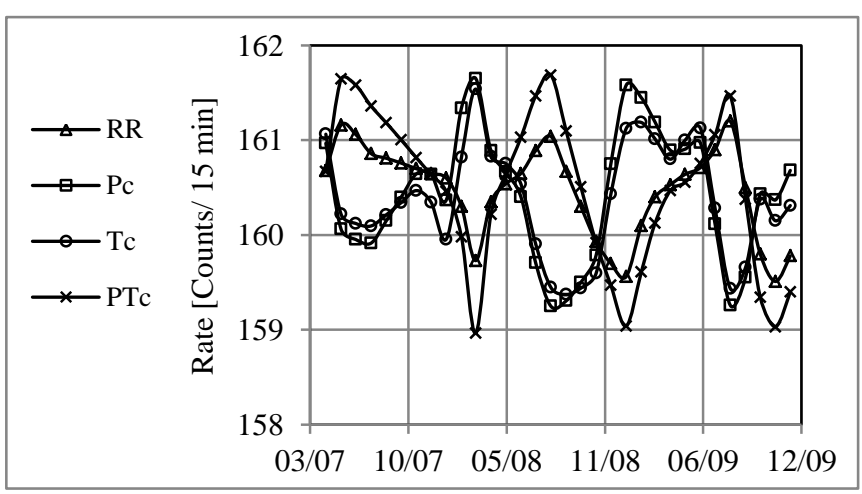

Figure (8). Time series of the one month average raw muon rate(RR), pressure corrected rates $(\mathrm{Pc})$, temperature corrected rate $(\mathrm{Tc})$, and pressure and temperature corrected rate $(\mathrm{PTc})$ rate for the period between 2007 and 2009.

\section{Conclusion}

In this study, the effect of atmospheric pressure, temperature, and relative humidity on cosmic ray muons has been investigated. The muon data were obtained using the KACST muon detector for the quiet solar period between 2007 and 2009. It was found that the pressure and temperature are anti-correlated with the muon rates. The obtained coefficients, particularly the temperature coefficient, are small and slightly different from those reported by several other investigators. This may be due to the dependence of these coefficients on factors such as the 
type of secondary particles and their energies. An acceptable positive correlation was found between the muon rates and the relative humidity. The magnitude of the relative humidity coefficient is comparable with the temperature coefficient. Multiple regression analyses have been performed between the muon rates and both the pressure and temperature; the desired coefficients have been obtained and used to remove the effects of these variables. All of the regression results obtained between the muon data and the considered variables have been tested and found to be statistically significant. Finally, although the influences of the three variables on the measured muon data have been corrected for, seasonal variations remain.

\section{Acknowledgments}

The authors would like to thank King Abdulaziz City for Science and Technology (KACST) for supporting this work.

\section{References}

[1] Kudela, K., 2016. On low energy cosmic rays and energetic particles near Earth, Contrib. Astron. Obs. Skalnate Pleso, 46, 15-70, 2016.

[2] Svensmark H., Friis-Christiansen E., Variation of cosmic ray flux and global cloud coverage - A missing link in solar-climate relationships. J. Atmos. Solar Terr. Phys. 59 1225-1232, 1997.

[3] Manginia A. et al.,, Evidence for a link between the flux of galactic cosmic rays and Earth's climate during the past 200,000 years", J. Atmos. Solar Terr. Phys.66 (3-4), 313-322, 2004.

[4]Dorman, L., 2004, Cosmic rays in the Earth's atmosphere and underground, Kluwer Academic Publishers, USA.

[5] Baker, P. et al., Atmospheric correction analysis for the Mawson muon telescopes. 23rd ICRC,Calgary, 3, 753,1993.

[6] DeMendonça R. et al., Analysis of atmospheric pressure and temperature effects on cosmic ray measurements . J. Geophys. Res118, 1403,2013.

[7] Maghrabi A.H., Almutayri M., Atmospheric Effect on Cosmic Ray muons at High Cutoff Rigidity Station, Advances in Astronomy, V. 2016, Article ID 9620189, 2016.

[8] Bchelet, F., et al., The cosmic-ray spectral modulation above 2 GV, Nuovo Cimento 11B, 1-12, 1972.

[9] Chilingarian A., et al., Calculation of the barometric coefficients for the particle detectors belonging to the world-wide networks at the start of the 24th Solar Activity cycle, 31 st ICRC,

ŁODZ. 2009

[10] Maghrabi A. et al., The KACST muon detector and its application to cosmic-ray variations studies, Advances in Space Research, 50, 6,700-711, 2012.

[11] Maghrab A. et al., On the calibration of a single channel cosmic ray particle detector, Proc. SPIE 9154, VI, 91542K, 2014.

[12] Maghrabi A. H., et al., Influence of the Atmospheric Mass on the High Energy Cosmic Ray Muons during a Solar Cycle, Advances in Astronomy doi.org/10.1155/2015/939146.

[13] Zazyan M., et al., Atmospheric effect corrections of MuSTAnG data . J. Space Weather Space Clim., $5, \mathrm{~A} 6,2015$.

[14] Kovylyaeva A . et al., 2013 , In Proc. of 23rd ECRS (and 32nd Russian Cosmic ray Conf. 409, 012128, 2013.

[15] Duperier A., The Temperature Effect on Cosmic-Ray Intensity and the Height of Meson Formation, Proc. Phys. Soc. 61 34, 1948. 